\title{
BI-PENALTY STABILIZED EXPLICIT FINITE ELEMENT ALGORITHM FOR ONE-DIMENSIONAL CONTACT-IMPACT PROBLEMS
}

\author{
R. Kolman ${ }^{*}$, J. Kopačka ${ }^{* *}$, A. Tkachuk ${ }^{* * *}$, D. Gabriel ${ }^{* * * *}$, J. A. González ${ }^{* * * * *}$
}

\begin{abstract}
In this contribution, a stabilization technique for finite element modelling of contact-impact problems based on the bipenalty method and the explicit predictor-corrector time integration is presented. The penalty method is a standard method for enforced contact constrains in dynamic problems. This method is easily implemented but the solution depends on numerical value of the stiffness penalty parameter and also the stability limit for explicit time integration is effected by a choice of this parameter. The bipenalty method is based on penalized not only stiffness term but also mass term concurrently. By this technique with a special ratio of mass and stiffness penalty parameters, the stability limit of contact-free problem is preserved. In this contribution, we also present a modification of the explicit time scheme based on predictor-corrector form. By meaning of this approach, spurious contact oscillations are eliminated and the results do not depend on numerical parameters.
\end{abstract}

Keywords: Finite element method, Contact-impact problems, Explicit time integration, Penalty and bipenalty methods.

\section{Introduction}

A numerical stabilization technique based on the finite element method for modelling of contact-impact problems is presented. Presently, penalty methods, Lagrange Multiplier methods or augmented Lagrangian methods are standard methods for modelling of dynamic contact problems in the finite element method, see Belytschko (2008). In this paper, the finite element method, see Hughes (2000)s with the penalty method for contact enforcing is employed. In explicit finite element analysis, the penalty method is preferred due to its simplicity, see Belytschko (2008). In the principle, the stiffness term is added into the strain energy in the variational formulation of a contact-impact problem. The classic penalty method has a disadvantage that the stable time step is attacked by the stiffness penalty parameter. Further, it is needed to set a suitable value of the stiffness penalty parameter and results depend on this value.

A modification called the bipenalty method is able to kept the stable time step size corresponding to contactfree problems independent of value of stiffness penalty parameter. In this approach, the inertia (mass) term is also penalized and the dynamical system corresponding to contact pairs can be controlled by the mass and stiffness penalty parameters simultaneously. With a special setting of ratio of mass and stiffness penalty parameters, the maximum eigen-frequency dictating the stability limit is preserving in comparison with contact-free problems. And the stiffness penalty parameter can be chosen arbitrarily. The application of the bipenalty method into dynamic conctact problems have been applied in Kopačka (2018).

Ing. Radek Kolman, Ph.D.: Institute of Thermomechanics, The Czech Academy of Sciences, Dolejškova 5; 182 00, Prague; CZ, kolman@it.cas.cz

** Ing. Ján Kopačka, Ph.D.: Institute of Thermomechanics, The Czech Academy of Sciences, Dolejškova 5; 182 00, Prague; CZ, kopacka@it.cas.cz

*** Dr. Anton Tkachuk: Institute for Structural Mechanics, University of Stuttgart; Pfaffenwaldring 7; 70550 Stuttgart; Germany, tkachuk@ibb.uni-stuttgart.de

***** Ing. Dušan Gabriel, Ph.D.: Institute of Thermomechanics, The Czech Academy of Sciences, Dolejškova 5; 182 00, Prague; CZ, gabriel@it.cas.cz

****** Prof. José A. González: Escuela Técnica Superior de Ingeniería, Universidad de Sevilla, Camino de los Descubrimientos s/n; E-41092 Seville; Spain, japerez@us.es 
Another trouble in modelling of dynamic contact problems is existence of spurious contact oscillations. In this paper, we suggest a methodology witch is able to avoid the both mentioned troubles in modelling of dynamic contact problems and we applied them into one-dimensional problems. We take together the bipenalty method, see Askes (2010); Hetherington (2013), and the predictor-corrector form, see Wu (2003), for explicit time integration of stabilized equation of motion with contact boundary conditions.

\section{Bipenalty method in finite element method for contact-impact problems}

In the work Kopačka (2018), the equations of motion of impacted elastic solids with respect to the bipenalty stabilization has been derived. Also the stability of the bipenalty method has been analyzed there. We shortly remark the basics of application of the finite element method for modelling of contact-impact problems in an one-dimensional case with bipenalty terms (additional penalized stiffness and mass terms, see Kopačka (2018)). Equations of motion for contact-impact problems under infinitesimal theory modelled by the finite element method are given by the following system of equations

$$
\mathbf{M} \ddot{\mathbf{u}}(t)+\mathbf{K u}(t)+\mathbf{R}_{\mathrm{c}}(\mathbf{u}, \ddot{\mathbf{u}})+\mathbf{R}(t)=\mathbf{0}
$$

where $\mathbf{u}, \dot{\mathbf{u}}, \ddot{\mathbf{u}}$ mark the global vectors of nodal displacements, velocities and accelerations, respectively. $\mathbf{M}$ is the global mass matrix, $\mathbf{K}$ is the global stiffness matrix, $\mathbf{R}(t)$ is the time-dependent global load vector, $t$ is the time. Finally, $\mathbf{R}_{\mathrm{c}}$ is the global contact residual vector given in the local elemental form as

$$
\hat{\mathbf{R}}_{\mathrm{c}}(\hat{\mathbf{u}}, \ddot{\hat{\mathbf{u}}})=\hat{\mathbf{M}}_{\mathrm{p}} \ddot{\hat{\mathbf{u}}}+\hat{\mathbf{K}}_{\mathrm{p}} \hat{\mathbf{u}}+\hat{\mathbf{f}}_{\mathrm{p}}
$$

where

$$
\hat{\mathbf{M}}_{\mathrm{p}}=\int_{\Gamma_{\mathrm{c}}} \epsilon_{\mathrm{m}} H(g) \mathbf{N N}^{\mathrm{T}} \mathrm{d} S \quad \hat{\mathbf{K}}_{\mathrm{p}}=\int_{\Gamma_{\mathrm{c}}} \epsilon_{\mathrm{s}} H(g) \mathbf{N} \mathbf{N}^{\mathrm{T}} \mathrm{d} S \quad \hat{\mathbf{f}}_{\mathrm{p}}=\int_{\Gamma_{\mathrm{c}}} \epsilon_{\mathrm{s}} H(g) \mathbf{N} g_{0} \mathrm{~d} S
$$

Here, $\hat{\mathbf{M}}_{\mathrm{p}}$ is the additional elemental mass matrix due to inertia penalty, $\hat{\mathbf{K}}_{\mathrm{p}}$ is the additional elemental stiffness matrix due to stiffness penalty, $\hat{\mathbf{u}}$ is the vector of displacements of contact pairs and $\hat{\mathbf{f}}_{\mathrm{p}}$ is the part of the elemental contact force due to the initial gap $g_{0} ; g$ is the gap function; $H(g)$ is the Heaviside step function for prescribing active or inactive contact constraints; $\epsilon_{\mathrm{m}}$ and $\epsilon_{\mathrm{s}}$ are mass and stiffness penalty parameters; $\Gamma_{\mathrm{c}}$ is the contact surface between bodies; the matrix $\mathbf{N}$ represents an operator from the displacement field $\mathbf{u}$ to the gap function $g_{\mathrm{N}}$ in the contact

$$
g_{\mathrm{N}}=\mathbf{N}^{\mathrm{T}} \mathbf{u}+g_{0} .
$$

The particular form of the matrix $\mathbf{N}$ follows from the used contact discretization. Note, that the matrices $\mathbf{M}_{\mathrm{p}}$ and $\mathbf{K}_{\mathrm{p}}$ are time-dependent because they are associated with active contact constrains.

\section{Discretized equations of motion with the bipenalty stabilization}

Now, we assume the time integration of the semi-discretized system (1) in the framework of the central difference method Belytschko (2008) as

$$
\left(\mathbf{M}^{t}+\mathbf{M}_{\mathrm{p}}^{t}\right) \frac{\mathbf{u}^{t+\Delta t}-2 \mathbf{u}^{t}+\mathbf{u}^{t-\Delta t}}{\Delta t^{2}}+\left(\mathbf{K}^{t}+\mathbf{K}_{\mathrm{p}}^{t}\right) \mathbf{u}^{t}+\mathbf{f}_{\mathrm{p}}^{t}-\mathbf{R}^{t}=\mathbf{0}
$$

Assuming that displacements are known at time $t-\Delta t$ and $t$, one can resolve unknown displacements at time $t+\Delta t$, where $\Delta t$ marks the time step size. We assume the diagonal mass matrix and linear shape functions.

\section{Stability analysis}

In the case of explicit time integration by the central difference method, the stability limit is given as $\Delta t \leq 2 / \omega_{\max }$, see Park (1977), where $\omega_{\max }$ is computed as the maximum eigen-value of $\left[-\omega^{2}(\mathbf{M}+\right.$ $\left.\left.\mathbf{M}_{\mathrm{p}}\right)+\left(\mathbf{K}+\mathbf{M}_{\mathrm{p}}\right)\right] \mathbf{U}=\mathbf{0}$. The stability analysis for bipenalized dynamical systems for different geometrical cases of contact problems in one-dimensional case has been studied in Kopačka (2018). For examples, the 


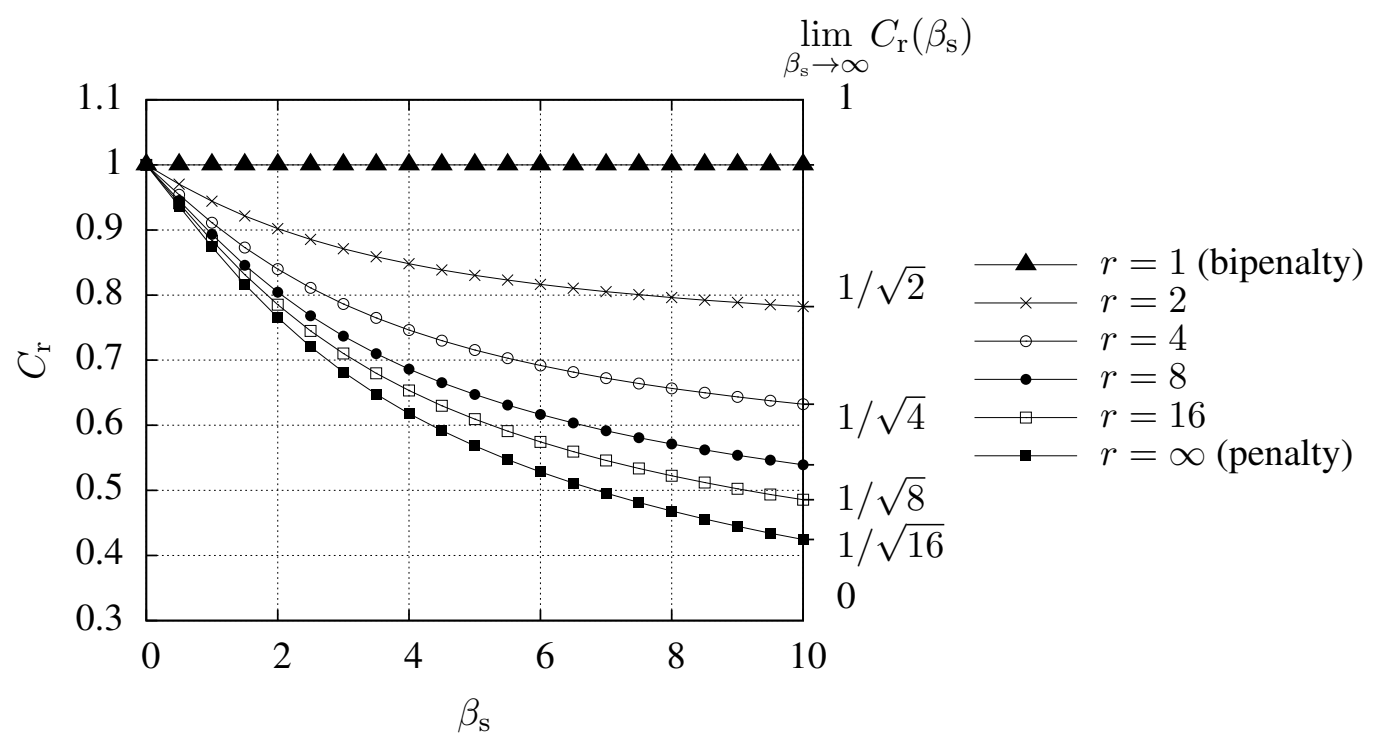

Fig. 1: Bipenalized Signorini problem: Dependence of the critical Courant number $C_{\mathrm{r}}$ on the dimensionless stiffness penalty $\beta_{\mathrm{s}}$ for selected ratios $r=\frac{1}{2} \frac{\beta_{s}}{\beta_{m}}$, see Kopačka (2018).

stability graph for dimensionless stiffness and mass penalty parameters $\left(\beta_{s}\right.$ and $\left.\beta_{m}\right)$ is shown in Fig. 1 for the Signorini problem. One can see the stability limit for the penalty method $(r=\infty)-$ the stability limit rapidly decreases with a larger value of stiffness penalty parameter. Based on this stability study, there exists a special setting of ratio of dimensionless stiffness and mass penalty parameters $(r=1)$ so that the stability limit for contact-free problems is preserved.

\section{Time integration of equation of motion with contact constrains}

The stabilized explicit time integration scheme for contact-impact problems is mentioned in depth. In the work $\mathrm{Wu}$ (2003), the fully explicit time integration scheme with stabilized technique for contact-impact problems has been published and tested. The mentioned time integration scheme takes the following flowchart with splitting bulk and contact accelerations for application with the bipenalty method:

- Given $\mathbf{u}^{t}, \dot{\mathbf{u}}^{t-\Delta t / 2}, \mathbf{f}_{\text {ext }}^{t}$

- Compute accelerations of predictor phase $\ddot{\mathbf{u}}_{\text {pred }}^{t}=\mathbf{M}^{-1}\left(\mathbf{f}_{\text {ext }}^{t}-\mathbf{K} \mathbf{u}^{t}\right)$

- Mid-point velocities of predictor phase $\dot{\mathbf{u}}_{\text {pred }}^{t+\Delta t / 2}=\dot{\mathbf{u}}^{t-\Delta t / 2}+\Delta t \ddot{\mathbf{u}}_{\text {pred }}^{t}$

- Displacements of predictor phase $\mathbf{u}_{\text {pred }}^{t+\Delta t}=\mathbf{u}^{t}+\Delta t \dot{\mathbf{u}}_{\text {pred }}^{t+\Delta t / 2}$

- For given $\mathbf{u}_{\text {pred }}^{t+\Delta t}$ analyze contact, compute gap vector $\mathbf{g}$ and contact forces $\mathbf{f}_{\text {cont }}=-\mathbf{K}_{\mathrm{p}} \mathbf{u}_{\text {pred }}^{t+\Delta t}+\mathbf{f}_{\mathrm{p}}^{0}$

- Compute accelerations of corrector phase $\ddot{\mathbf{u}}_{\text {corr }}^{t}=\left(\mathbf{M}+\mathbf{M}_{\mathrm{p}}\right)^{-1}\left(\mathbf{f}_{\text {cont }}\right)$

- Compute total accelerations $\ddot{\mathbf{u}}^{t}=\ddot{\mathbf{u}}_{\text {pred }}^{t}+\ddot{\mathbf{u}}_{c o r r}^{t}$

- Mid-point velocities of corrector phase $\dot{\mathbf{u}}^{t+\Delta t / 2}=\dot{\mathbf{u}}_{\text {pred }}^{t+\Delta t / 2}+\Delta t \ddot{\mathbf{u}}_{\text {corr }}^{t}$

- New displacements of corrector phase $\mathbf{u}^{t+\Delta t}=\mathbf{u}^{t}+\Delta t \dot{\mathbf{u}}^{t+\Delta t / 2}$

- For given $\mathbf{u}^{t+\Delta t}$ analyze contact, compute gap vector $\mathbf{g}$ and contact forces $\mathbf{f}_{\text {cont }}^{t+\Delta t}=-\mathbf{K}_{\mathrm{p}} \mathbf{u}^{t+\Delta t}+\mathbf{f}_{\mathrm{p}}^{0}$

- $t \rightarrow t+\Delta t$

In this two-time step scheme, bulk accelerations in the predictor phase $\ddot{\mathbf{u}}_{\text {pred }}^{t}$ are computed only for internal and external forces without information about contact constrains. After updating of velocities and displacements, contact constraints are analyzed and contact forces $\mathbf{f}_{\text {cont }}$ are evaluated. For these contact forces, contact accelerations in the corrector phase $\ddot{\mathbf{u}}_{\text {corr }}^{t}$ are computed with the additional penalized mass matrix $\mathbf{M}_{\mathrm{p}}$. After that, the both parts of accelerations are taken together.

The suggested methodology is possible to extent for multi-dimensional problems in the same sense with correct evaluation of penalized matrices, and also into fully geometrical and material non-linear problems where one has to use correct evaluation of internal forces for nonlinear problems (e.g. in connection with consistent co-rotational formulation) and with updating of actual spatial coordinates, see Belytschko (2008). 


\section{Numerical benchmark - impact of bars}

As a numerical test, we study a contact-impact problem of two elastic bars with different lengths defined in work of Huněk (1993) and for scheme see Fig. 2 left. The left bar is moving to the right with a constant velocity and the right bar with fixed right-hand side is at rest. In Fig. 2 right, one can see the time history of contact force between two elastic bars computed by the stabilized explicit scheme and the bipenalty method. The results exhibit promising physically correct results.
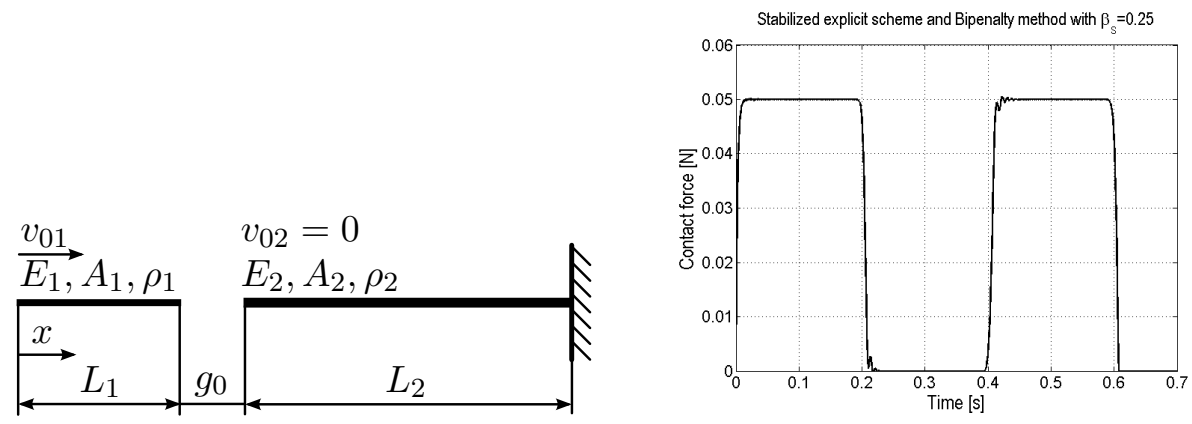

Fig. 2: A scheme of an one-dimensional impact of two bars with different lengths (on the left). Time history of contact force for impact of two bars with different lengths - the stabilized explicit method with the Courant number $C=0.5$ for dimensionless numerical stiffness penalty parameter $\beta_{s}=0.25$ and optimal bipenalty stabilization setting (on the right).

\section{Conclusions}

In this contribution, we have suggested the methodology for finite element modelling of contact-impact problems. The method has been tested for an one-dimensional problems. The method produces superior properties. In the future, we plan testing numerical stability and sensitivity on numerical parameters in depth and to extent the method for multi-dimensional problems. In this case, the implementation is the same as in one-dimenstional cases.

\section{Acknowledgments}

The work of Radek Kolman was supported by the Grant project with No. 19-04956S of the Czech Science Foundation (CSF) within institutional support RVO:61388998. The work of Ján Kopačka was supported by the Grant project with No. 19-14237S of CSF. The work of Dušan Gabriel was supported by the Centre of Excellence for Nonlinear Dynamic Behaviour of Advanced Materials in Engineering CZ.02.1.01/0.0/0.0/15_003/0000493 (Excellent Research Teams) in the framework of Operational Programme Research, Development and Education.

\section{References}

Askes, H., Caramés-Saddler, M., Rodríguez-Ferran, A (2010) Bipenalty method for time domain computational dynamics. Proc. Royal. Soc. A., 466, pp 1389-1408.

Belytschko, T., Liu, W.K., Moran, B. (2008) Nonlinear finite elements for continua and structures, the first edition. Wiley.

Hetherington, J., Rodríguez-Ferran, A., Askes, H (2013) The bipenalty method for arbitrary multipoint constraints. Int J Numer Methods Eng., 93: pp 465-482.

Hughes, T.J.R. (2000) The Finite Element Method: Linear and Dynamic Finite Element Analysis. Dover Publications: New York.

Huněk, I. (1993) On a penalty formulation for contact-impact problems. Comput Struct. 1993; 48(2), pp 193-203.

Kopačka, J., Tkachuk, A., Gabriel, D., Kolman, R., Bischoff, M., Plešek, J. (2018) On stability and reflectiontransmission analysis of the bipenalty method in impact-contact problems: a one-dimensional, homogeneous case study, Int J Numer Methods Eng., 113(10), pp 1607-1629.

Park, K.C. (1977) Practical aspect of numerical time integration. Computures \& Structures, 7, pp 343-353.

Wu, A.R. (2003) A priori error estimates for explicit finite element for linear elasto-dynamics by Galerkin method and central difference method. Comput Methods Appl Mech Eng. 192(81), pp 5329-5353. 